Orinoquia, Enero-Junio 2020;24(1): 86-93

ISSN electrónico 2011-2629.

ISSN impreso 0121-3709.

http://doi.org/10.22579/20112629.597

\title{
Caño Gramalote: Historia de un siniestro
}

\author{
Caño Gramalote: a sinister story
}

Caño Gramalote: História de um sinistro

Hernando Castro-Garzón ${ }^{1}$; Francy L Montealegre-Torres ${ }^{2}$

1 AEA, Esp, MSc, PhD, Profesor Ocasional de la Universidad de los Llanos de la Facultad de Ciencias Económicas en el programa de Administración de Empresas. Villavicencio, Colombia.

$2 \mathrm{AE},(\mathrm{c}) \mathrm{Msc}$, Maestranta en Gestión de Desarrollo Sustentable Universidad Autónoma de Guerrero. Acapulco, México

Email: hcastro@unillanos.edu.co

Recibido: 23 de agosto de 2019

Aceptado: 23 de marzo de 2020

\section{Resumen}

A través de un estudio tipo mixto de orden cualitativo, aplicado a los residentes del barrio Camilo Torres en la rivera del caño Gramalote en el municipio de Villavicencio, Meta, Colombia, se determinó la vulnerabilidad de los predios y sus afectaciones por inundación; se cruzó lo observado con los referentes secundarios para determinar las acciones realizadas frente a los eventos ocurridos en la zona. Se utilizó la estrategia "historia de vida" para la recolección de información, construyendo un cuerpo textual para establecer relaciones y significados. Determinando que el cambio en el uso del suelo tanto como las medidas de contención adoptadas por la municipalidad han generado un aumento en la vulnerabilidad de la población allí residente.

Palabras Clave: Riesgo; territorio; desastre; sostenible; inundación

\begin{abstract}
Abstrac
This was a qualitative study of residents living in the Camilo Torres neighbourhood on the riverbanks of Caño Gramalote in the municipality of Villavicencio, Meta, Colombia; it involved providing illustrative mixed-method examples regarding the properties' vulnerability and determined the effects of flooding. What was physically observed was crossed with secondary references to determine action taken against events in the area. The "life history" strategy was used for gathering information from which a body of text was constructed for establishing relationships and meaning. This determined that changes in land use along with the containment measures taken by the municipality have led to the increased vulnerability of the population residing there.
\end{abstract}

Key words: risk; territory; disaster; sustainability; flooding.

\section{Resumo}

Por meio de um estudo qualitativo de tipo misto, aplicado aos moradores do bairro Camilo Torres, nas margens do Caño Gramalote, no município de Villavicencio, Meta, Colômbia, foi determinada a vulnerabilidade dos prédios e as afetações 
por inundação; o que foi comparado com os referentes secundários para determinar as ações realizadas para enfrentar os eventos que ocorreram na área. A estratégia da "história de vida" foi usada para coletar informações, construindo um corpo textual para estabelecer relacionamentos e significados. Determinando que a mudança no uso da terra e as medidas de contenção adotadas pelo município geraram um aumento na vulnerabilidade da população residente.

Palavras-chave: Risco; território; desastre; sustentável; inundação

\section{Introducción}

La ciudad de Villavicencio es un enclave colonial, lugar obligado de paso hacia los Llanos Orientales y la región de la selva; fue fundada hace más de 140 años (Rausch, 2011), no quiere decir esto que no fuere poblada desde mucho antes con diversos nombres según fuere el momento del asentamiento humano; ya que fue ubicada al lado de la cordillera oriental de los Andes colombianos como lugar estratégico para el comercio con las tierras llanas de sabana conexas, las selvas y la ciudad de Bogotá como capital del país; en esta génesis la fundación fue realizada principalmente por personas provenientes de municipios como Cáqueza, Choachi, Chipaque, Guayabetal, todos estos ubicados en la cordillera Andina y pertenecientes al departamento de Cundinamarca; lo anterior para significar la tendencia cultural hacia las costumbres de organización basados en el contexto alto andino que se modifica en el piedemonte llanero (Ibíd.).

Por lo descrito reglones atrás Villavicencio tiene unas particularidades en cuanto a la distribución urbana que la constriñe por más de 120 años a un centro urbano histórico y desde allí inicia su crecimiento (POT, 2015); siendo éste realizado de manera desordenada en especial en las laderas de sus ríos, por su característica de piedemonte fluyen varios por la ciudad, y que fueron ocupadas por población de escasos recursos económicos y con restricciones en términos de calidad de vida.

Este trabajo tiene como objeto principal analizar la percepción de vulnerabilidad y riesgo en relación con el caño Gramalote en el sector Camilo Torres frente a los eventos de inundación de dicho afluente. Donde se inicia con una descripción epistemológica y conceptual de los términos: vulnerabilidad, riesgo e inundación para de esta manera observar la afectación causada; seguidamente se contextualizará sobre el caño Gramalote y su afectación; para lo cual se usó la técnica de investigación cualitativa historia de vida "examinar la trayectoria de una sola persona, a partir de lo que dice él mismo, su familia y las personas que trabajan directamente con él" (Borboa, 2012) con un afectado por los hechos de inundación acaecidos.

\section{Materiales y métodos}

Se realizó una investigación tipo mixta; ya que se iniciará con un método exploratorio-descriptivo, consistente en identificar los predios ubicados en la ribera del caño Gramalote en el sector Camilo Torres, donde se estableció su vulnerabilidad. Luego se abordaron algunos predios para comprender su afectación frente a la información existente. También se aplicará el método inductivo para el análisis de casos, documentos y expedientes, con el propósito de comparar e identificar como se han abordado las situaciones que han generado siniestro y cuál ha sido la respuesta de las instituciones con los poseedores o dueños de los predios; para así, extraer conclusiones, proponer recomendaciones y de alguna manera incidir en el fortalecimiento social e institucional en el abordaje de los riesgos encontrados.

Se realizó la estrategia de historia de vida "unidad narrativa, temporal y discontinua que un sujeto hace acerca de sí. La misma consiste en una reconstrucción de acontecimientos y supone su conexión con otros acontecimientos pasados y presentes así como también con respecto a posibles eventos del futuro" (Leite, 2011) como método cualitativo, para establecer contacto con un integrante de la comunidad en aras de conocer su experiencia (Patton, 1980) sobre los eventos presentados en el Caño Gramalote.

Para el tratamiento analítico de la información se apunta al uso de conceptos en contraste con el objeto de estudio, creando un cuerpo textual para establecer relaciones entre sus diferentes componentes; posteriormente, también se incluye un registro de información para la posterior síntesis del material empírico colectado; adicionalmente se entramó a partir de la interpretación para determinar significados y comprenderlos a partir de la información obtenida.

\section{Resultados}

\section{Conceptualización}

La vulnerabilidad vista desde la perspectiva geográfica es la "disposición interna a ser afectado por una amenaza.... Depende del grado de exposición, de la protección, de la reacción inmediata, de la recuperación 
básica y de la reconstrucción" (IGUNNE. 2010); desde lo social "inseguridad e indefensión que experimentan las comunidades, familias e individuos en sus condiciones de vida a consecuencia del impacto provocado por algún tipo de evento económico social de carácter traumático" (CEPAL, 2001).

Se observa así como la vulnerabilidad se aplica como concepto multidimensional, que tan solo es un término aplicable a los factores de exposición humana; sean estos, económicos, religiosos, culturales, políticos, geográficos y finalmente en la gestión del desastre como lo permite observar la Federación Internacional de Sociedades de la Cruz Roja y la media Luna Roja:

"La exposición de las personas a riesgos varía en función de su grupo social, sexo, origen étnico u otra identidad, edad y otros factores. Por otra parte, la vulnerabilidad puede adoptar diferentes formas: la pobreza, p. ej., puede resultar en que las viviendas no puedan resistir a un terremoto o huracán, y la falta de preparación puede dar lugar a una respuesta más lenta al desastre, y con ello a más muertes o a un sufrimiento más prolongado".

El riesgo es otro término de interés para lograr entender el prisma que aquí se abarca, según (UNISDR, 2009) "el riesgo es la combinación de la probabilidad de que se produzca un evento y sus consecuencias negativas", de esta manera se observa que la naturaleza de la acción es latente y permanente, es un concepto amplio y profundo que no da cabida a especulaciones en cuanto a las consecuencias de este.

Por otro lado el Centro Internacional para la Investigación del Fenómeno de El Niño (CIIFEN, 2015) plantea el siguiente concepto frente al riesgo:" se define como la combinación de la probabilidad de que se produzca un evento y sus consecuencias negativas. Los factores que lo componen son la amenaza y la vulnerabilidad". De esta manera el riesgo puede ser causado por fenómenos de carácter natural; (Cardona, 1991) "Algunos desastres de origen natural corresponden a amenazas que no pueden ser neutralizadas debido a que difícilmente su mecanismo de origen puede ser intervenido, aunque en algunos casos puede controlarse parcialmente".

Así cuando el riesgo es tomado como una probabilidad tiene una connotación matemática en la cual se considera el seguimiento, monitoreo y predictibilidad de los fenómenos naturales reduciendo considerablemente y preparando de esta manera a las diferentes entidades encargadas de estos eventos, y por supuesto reduciendo el número de vidas perdidas así lo permite observar UNISDR (2009):

\section{Riesgo $=$ Amenaza $X$ Vulnerabilidad}

Los factores que componen la vulnerabilidad son la exposición, susceptibilidad y resiliencia, expresando su relación en la siguiente fórmula:

$$
\begin{aligned}
\text { Vulnerabilidad }=\underset{\text { Resiliencia }}{\text { Exposición X Susceptibilidad } /} \\
\text { Resil }
\end{aligned}
$$

Exposición es la condición de desventaja debido a la ubicación, posición o localización de un sujeto, objeto o sistema expuesto al riesgo.

Susceptibilidad es el grado de fragilidad interna de un sujeto, objeto o sistema para enfrentar una amenaza y recibir un posible impacto debido a la ocurrencia de un evento adverso.

Resiliencia es la capacidad de un sistema, comunidad o sociedad expuestos a una amenaza para resistir, absorber, adaptarse y recuperarse de sus efectos de manera oportuna y eficaz, lo que incluye la preservación y la restauración de sus estructuras y funciones básicas.

Con esta fórmula los gobiernos tienen un instrumento con destino a la atención de las emergencias a las alertas y por supuesto al diseño de tácticas para el restablecimiento, la restauración y la redención de las zonas afectadas; pero Ferrari (2012) argumenta frente a este método de previsión "La diversidad de percepciones existentes sobre un mismo problema ha originado en los estudios de riesgo una polémica en torno a las discrepancias entre los juicios de valor emitidos por los expertos y los sostenidos por otros actores sociales no expertos " ahora bien, dichos avances carecen de una estructura completa por lo cual no son suficientes para definir y mucho menos para darle un punto final al riesgo, ya que el crecimiento y expansión poblacional se han vuelto incontrolables para las entidades territoriales, así lo demuestra Da Cunha y Rodríguez (2009):

El crecimiento urbano se desaceleró por el avance de la transición demográfica y la merma del impacto cuantitativo de la emigración del campo sobre la expansión de la población urbana. Con todo, el éxodo rural ha continuado y con éste la urbanización, llevando a la región a índices del 80 por ciento de población urbana en la actualidad, solo superada por América del Norte

Y, Blaikie, Cannon, Davis y Wisner (1996) en mención a ello determinan:

La población vive en situaciones económicas adversas que la llevan a habitar partes del mundo que se ven afectadas por amenazas naturales, sean áreas de inundación de los ríos, laderas de volcanes o zonas sísmicas. Pero 
hay muchos otros factores políticos y económicos menos obvios que están tras el impacto de las amenazas.

Y finalmente (Alfie y Castillo, 2016) sintetiza en unas breves palabras su concepto frente al riesgo: "el riesgo corresponde al grado de pérdidas o daños esperados debido a la ocurrencia de un fenómeno natural o tecnológico peligroso" todo esta visión tiene arraigo en un eje humano, debido a las características de una población menos favorecida, por la exposición a la situación de vulnerabilidad frente a fenómenos naturales.

Teniendo los conceptos previos emitidos por las entidades pertinentes y responsables de los procesos de atención en cuanto a vulnerabilidad y riesgo, se tomara la inundación como objeto de estudio, donde (Faber, 2014) la define como:

Se trata de una situación en la cual el agua cubre un terreno que normalmente no está cubierto de agua; en pocas palabras, el agua se desborda o cubre tierra que suele estar seca. Las inundaciones pueden ser: a) locales, cuando afectan una comunidad o área pequeña y b) de afectación extensa, que pueden abarcar cuencas fluviales enteras y varias comunidades.

Es de anotar que el fenómeno de la inundación ha sido una constante en el territorio colombiano (Sedano, et al., 2013), ancestralmente fueron usadas como desarrollo agrícola; en la actualidad debido al crecimiento urbano desmesurado (Torres y Trujillo, 2014) al ser ubicado este en zonas de protección de causes o de recarga hídrica genera riesgos, amenazas y desastres.

De igual forma el incremento en las lluvias en los últimos 50 años ha contribuido a que las inundaciones sean aún más fuertes ya que con los fenómenos conocidos como el Niño y la Niña los cuales se repiten en una periodicidad de 4 a 7 años alternándose produciendo lluvias y sequías respectivamente aumentando la amenaza, Trenberth y Stepaniak, (2001) los describen como: "La Niña se caracteriza por temperaturas bajas y duraderas en el Océano Pacífico, mientras El Niño presenta temperaturas más elevadas a las normales. Ambos fenómenos producen cambios en el patrón de vientos atmosféricos sobre el Océano Pacífico tropical" al presentarse estos fenómenos alternos la vulnerabilidad es creciente ya que por la estacionalidad de periodos intermedios se ocupan terrenos en los periodos secos, sea como depósito de desechos o como terrenos de construcción, y al presentarse el fenómeno contrario se recargan las zonas presentando desastres por inundación. El alejarse de las costumbres tradicionales frente al conocimiento y comportamiento del territorio se acrecienta la amenaza por riesgos naturales.

\section{Área de estudio}

A partir de los cincuenta Colombia comenzó a tener un crecimiento acelerado de su población y con ello la masificación de las ciudades sin previos planes que permitieran la organización del territorio (Cohen, 2006) para así determinar los lugares adecuados para la urbanización.

Las estadísticas muestran que la ocurrencia de desastres naturales en países en vías de desarrollo ha aumentado significativamente en los últimos cincuenta años (BM, 2012). Dado que el peligro permanece más o menos constante, la explicación tiene que encontrarse en el hecho de que las condiciones de vulnerabilidad de la población y sus asentamientos están empeorando aceleradamente. (Llanes, 2003) "es así como en los últimos años se ha presentado emergencias por inundación y deslizamientos en las zonas cercanas a corrientes naturales de agua-humedales, ríos, caños, quebradas- ya que las edificaciones anteriormente nombradas obstruyen gran parte de dichas corrientes" Villavicencio siendo hoy en día una ciudad intermedia no es ajena a estos fenómenos puesto que sus condiciones geográficas e hidrográficas son la combinación perfecta para la ocurrencia de desastres (BANREP, 2000).

Villavicencio cuenta con una amplia riqueza hídrica ya que entre los principales afluentes que riegan su área de influencia están los ríos: Guatiquía, Guayuriba, Negro y Ocoa, así mismo los caños Parrado, Gramalote, Maizaro, Quebrada La Unión, Grande, Quebrada Honda, Buque, Rosablanca y La Cuerera (Cormararena, 2008). El caño Gramalote es el afluente hídrico que divide en dos la ciudad de Villavicencio, dicho caño nace en el cerro de Cristo Rey -elevación orográfica adyacente al centro de la ciudad- recorre los barrios: Mesetas, las Colinas, Azotea, Libertadores, San José, Camilo Torres, Centro, San Fernando, comuna 3; Porvenir, Industrial, Santa Fe, y comuna 4; Santa Helena, Jordan y Brisas del Guatiquía, para finalmente desembocar en el rio Guatiquía (ibíd.).

Debido a los efectos ocurridos por la inundación causada por el caño Gramalote el pasado 29 de abril de 2016 en el cual se presentaron "múltiples pérdidas materiales, histeria pública y diversos accidentes, uno de los habitantes del sector del barrio Camilo Torres sector de mayor afectación por la inundación" (El Tiempo, 2016). Se presenta la sensación de un poblador a partir del diálogo generado a manera de historia de vida a cerca de las percepciones frente a la situación de riesgo en su localidad. 


\section{RECORRIDO CAÑO GRAMALOTE VILLAVICENCIO META}

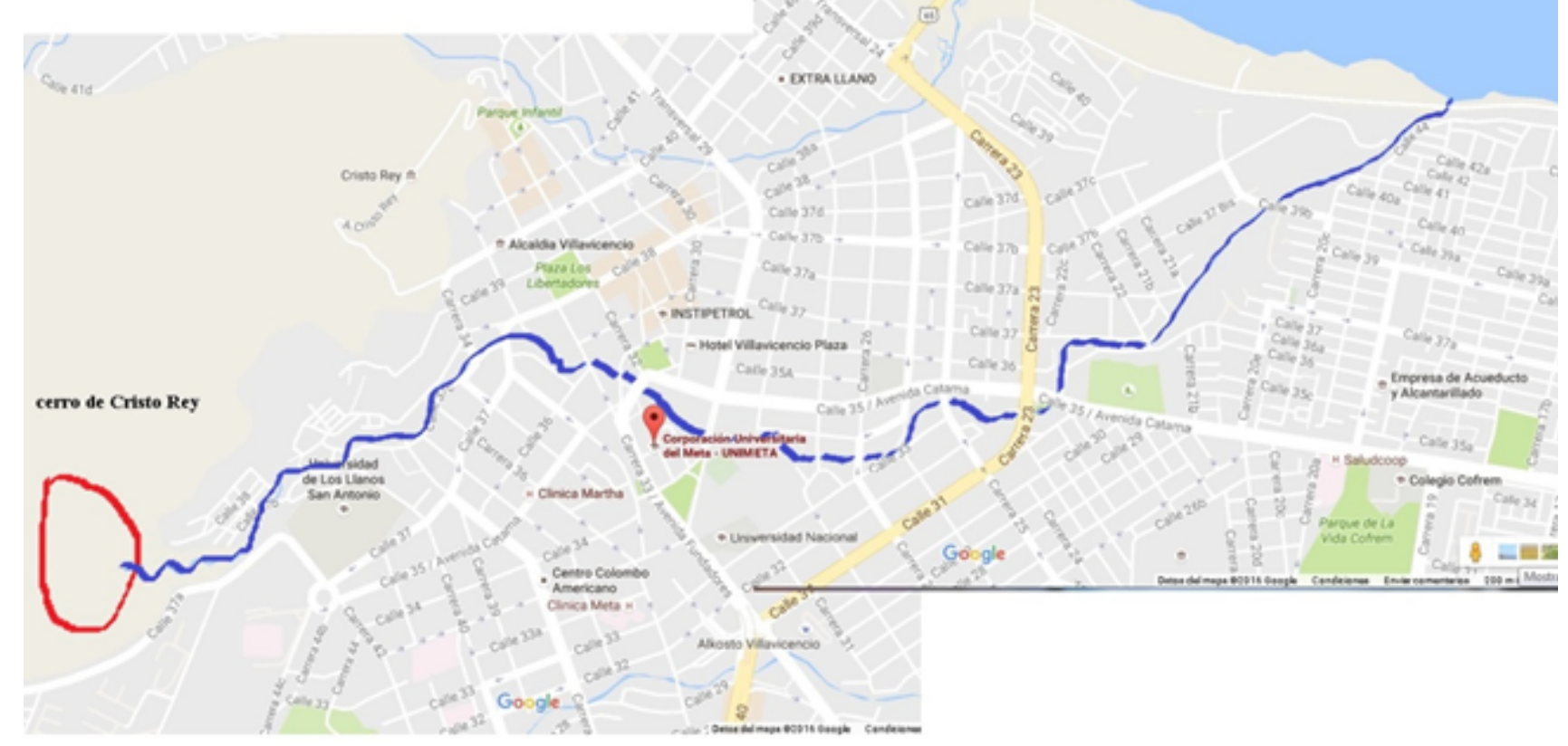

Figura 1. Recorrido Caño Gramalote

La situación más reciente de inundación y desbordamiento del caño Gramalote se dio el día 29 de abril del año 2016, "No habíamos tenido situaciones de estas tan riesgosas, si en la parte de arriba no hubieran botado basura, allá lo cogieron de botadero, los carros recolectores no pasaban, la botaban al caño." De esta manera el ciudadano inicia su relato, antes de ello da a conocer que habita el barrio Camilo Torres, hace ya algo más de dos años, su núcleo familiar está compuesto por su esposa y él, la vivienda que habitan está fabricada en ladrillo; cuando el informante enuncia - si en la parte de arriba- hace referencia al barrio San José donde se vive una situación de conflicto por bandas criminales y de micro tráfico (Alcaldía de Villavicencio, 2016); así continua la narración "Se hizo una concientización con la gente de San José, después de lo que pasó que se rebozó el caño. La Cruz se llenó, parecía una piscina, siempre ha estado en la parte baja. Eso tenía uno con cincuenta o dos metros hasta que se fue desocupando. Entonces concientizamos a la gente de arriba para que no volviera a botar las basuras al caño, porque qué pasó, ese día como hubo tanta agua y ahí abajo eso es muy pequeñito -hace referencia a la canalización del caño la cual tiene una medida de $150 \mathrm{~cm}$ de ancho por $200 \mathrm{~cm}$ de altura- entonces se represaron las basuras, al represarse las basuras y aquí se desbordó todo eso. Vivo hace dos años. Para nosotros eso era una cuestión de riesgo pero no teníamos el cómo llegar y hablar con esa gente de lo que nos pasó. Tuvo que haber pasado esto para que nosotros los hiciéramos caer en cuenta". Continua refiriéndose a las consecuencias para su criterio. "El agua se nos entró ese día como unos diez centímetros, -la construcción está divida en aparta estudios- y allá fueron más, pero no pasó a mayores. Ahora, no es riesgoso vivir aquí al lado de un cañito, siempre y cuando se tenga la conciencia de no tirar basuras".

"Yo creo que ya ahorita las lluvias que han pasado, el cañito no pasa de la mitad, ya baja con un caudal fuerte pero digamos que no alcanza a rebosar como ese día no". Se indaga sobre la participación de los entes institucionales, alcaldía o Gobernación, sobre la prevención del riesgo, a lo cual la respuesta es: "Lo estaban haciendo con el alcalde anterior, pero salió y ya quedó olvidado porque no hay plata. Él hizo el parque, con la construcción estaban haciendo el estudio porque pensaba canalizar más el caño para profundizarlo, pero sólo se llegó a un estudio no se analizó más eso a fondo." frente a la respuesta se indaga si conoce que es el riesgo a lo cual la respuesta fue: "El riesgo digamos es el tener conciencia de que estamos en una parte en donde puede ocurrir un desastre natural".

¿Se siente seguro en su casa? Con una contundente afirmacion dio respuesta y se hace el cuestionamiento frente a si se encuentran preparados para un desastre, y la respuesta fue "Digamos que eso muchas veces 
no lo tenemos en cuenta hasta que pasa algo." hasta cuando pasa la cuestión es que tenemos en cuenta que necesitamos irnos a una parte alta. Aquí tenemos la vía a la Salle, que es la entrada a Cristo Rey, sería la única que habría para evacuación"

Frente a la disposición de los residuos sólidos hace énfasis en que el vehículo recolector presta el servicio cada tercer día y la evacuación de aguas negras al servicio de alcantarillado y que poseen acueducto.

\section{Discusión}

A partir de la identificación de las "expresiones" (Eraso et al., 2012) brindadas por el informante permite reconocer historias de hechos sucedidos que cuentan experiencias subjetivas, en donde aparecen en los relatos relaciones directas con las percepciones de vulnerabilidad, riesgo e inundación. Desde la narrativa se constituyen y tejen formas que muestran sucesos vividos o argumentados; ya que estos desde el entendido de ser constructos mentales, por ende emocionales singulares y subjetivos, se configuran en una narración expresando la experiencia adquirida y por ende compartida; convirtiéndose en un generador de relaciones ya que los demás actúan de acuerdo a lo significante que ha sido para cada individuo.

Así las cosas el significado de "lo que hacen arriba" se constituye en un descriptor de amenaza que se establece en una variable de la condición de riesgo; la presunción de vulnerabilidad se plantea a partir de la significación de la cercanía al sistema de canalización del caño y la consideración de "es muy pequeñito" crea el sentimiento de aumento del riesgo por la ubicuidad de la vivienda.

Elementos como la susceptibilidad se dan en torno a las capacidades del sistema de canalización de soportar los volúmenes de agua retenidos por las disposiciones de basura; pero de allí nace un mayor significado frente al componente de resiliencia que a pesar de los hechos y de los posibles responsables se determina como creciente ya que se establece que la inundación genero cohesión entre la comunidad para disminuir la vulnerabilidad.

Frente a la presunción de seguridad la afirmación categórica establece que hay un enraizamiento al territorio; que a pesar de los hechos ocurridos la certidumbre de permanencia es alta aún con el reconocimiento de la posibilidad de un desastre "eso muchas veces no lo tenemos en cuenta" y se enuncia posibilidades de evacuación como solución a un evento catastrófico pero en ningún momento se determinan significados frente a la reubicación.

La posición frente a la institucionalidad es dividida ya que se pronuncia frente al abandono de la misma en cuanto a prevención pero enuncia que se realizaron obras públicas pero que son insuficientes en su capacidad de contención.

\section{Conclusiones}

El cambio en el uso de los suelos ha incrementado el riesgo por inundación; ya que urbanizar las zonas de protección de ronda de afluentes hace que aumente la vulnerabilidad y susceptibilidad al mismo.

La canalización del caño Gramalote posee dimensiones muy pequeñas para el flujo de agua en temporada de lluvias y menos aún para soportar represamientos ya que tiene medidas de 2,5 metros de profundidad por tan solo 1.5 de ancho.

La costumbre de tipo constructiva de canalizar afluentes no es una solución adecuada para los cuerpos de agua ya que no permiten la recarga hídrica ni los resaltos hídricos determinantes como servicio ecosistémico de regulación.

La densidad poblacional de este sector es muy alta, pero desafortunadamente previa a los planes de ordenamiento territorial, generando un conflicto entre la población y la administración del riesgo en la ciudad.

El establecimiento y abastecimiento de servicios públicos a estas zonas ha permitido una legalización de las mismas por tanto se cuenta indirectamente con la aprobación de este tipo de urbanización generando unas responsabilidades concretas a la municipalidad.

Adicionalmente a la presencia de amenaza natural en la zona se presenta un fenómeno social de violencia que incrementa la desestabilización de la población ya que no permite la intervención de personal de apoyo y prevención así como de la adecuada cohesión de los pobladores de dicha zona.

Para la administración municipal la reubicación de la zona afectada es la solución; pero el costo económico y político no ha permitido una adecuada gestión en cuanto al cumplimiento de la normativa en referencia a las zonas de protección de caños y ríos.

Las poblaciones asentadas en esas riveras presentan alto grado de resiliencia frente a las inundaciones presentadas. 
Una razón adicional para que este evento haya ocurrido fue el cambio del uso del suelo, ya que como lo da a conocer Carvajal (2011) "El principal objetivo del drenaje urbano es evacuar rápidamente excesos de escorrentía superficial, lo cual contribuye a incrementar los picos de crecidas y a reducir tiempos de concentración" de esta manera la acumulación de sedimento y desechos se incrementa tanto en el cauce como en los canales reduciendo la capacidad de conducción hídrica del canal.

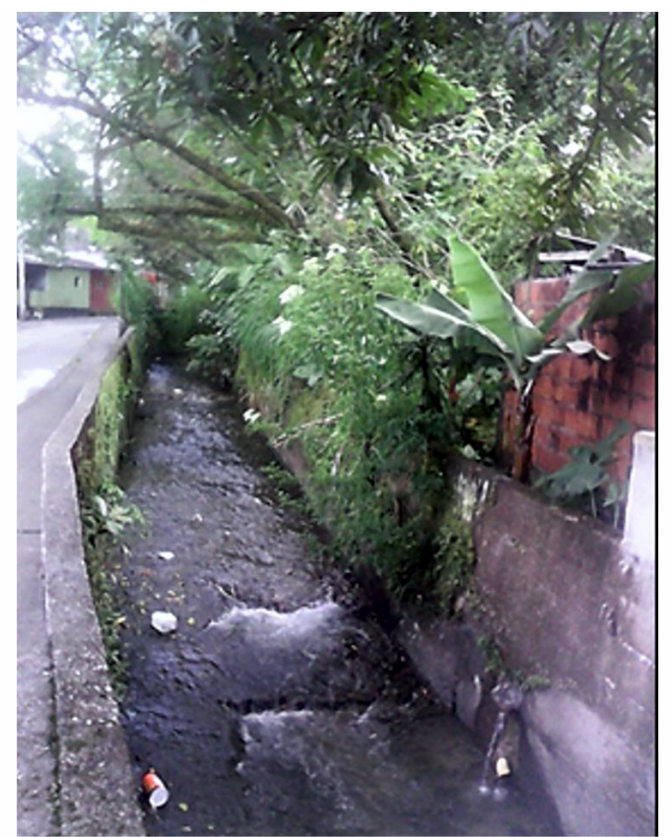

\section{Recomendaciones}

Para el restablecimiento de áreas de ronda de protección es necesario tener en cuenta los comportamientos históricos de los afluentes para establecer una adecuada gestión del riesgo.

Son necesarias políticas de implementación de procesos de reubicación y aprendizaje consensuado de las comunidades insertas o conexas a las zonas de amenazas; esto con el fin de crear dinámicas que eviten las pérdidas materiales y humanas.
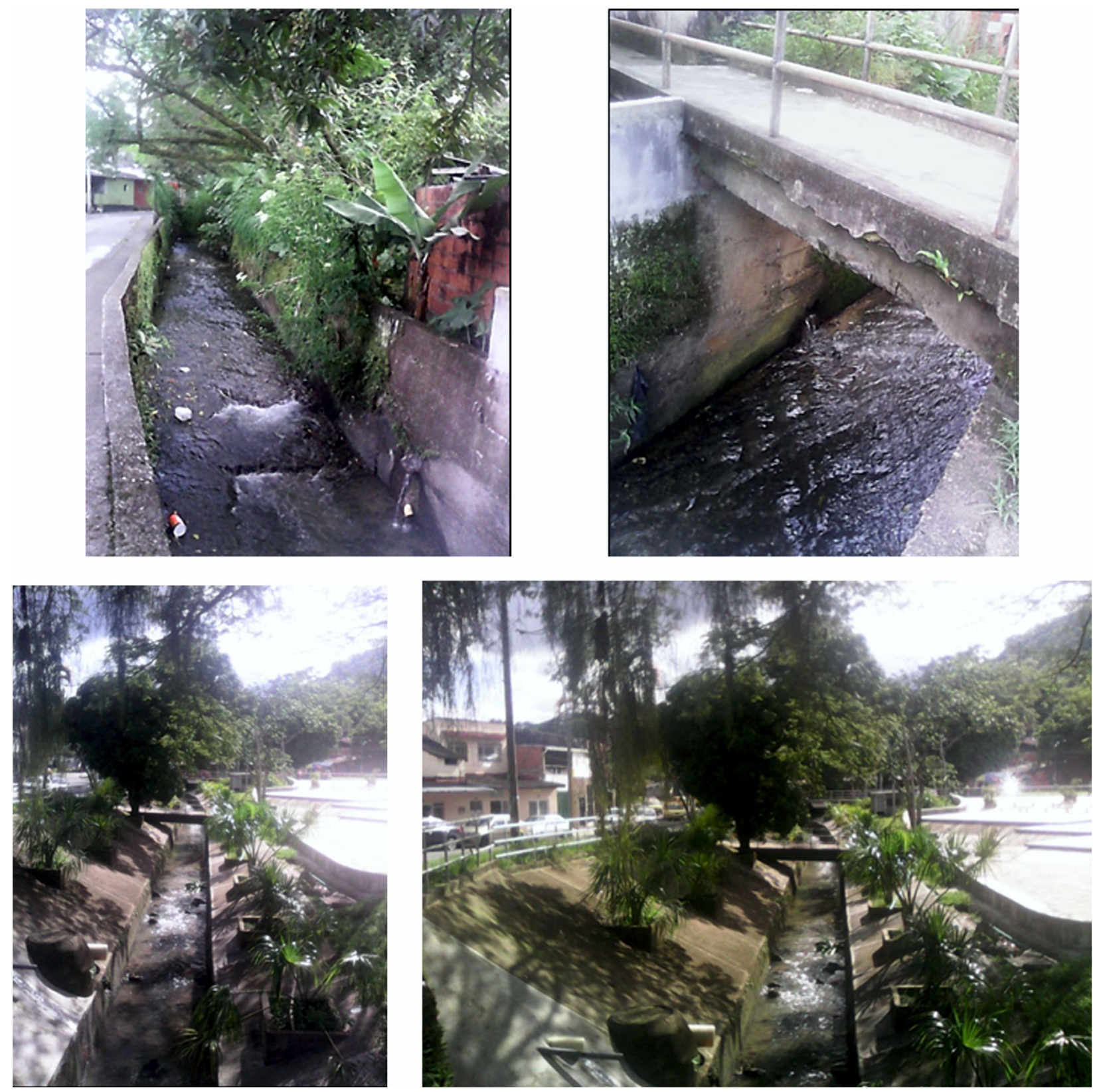

Figura 2. Imágenes Caño Gramalote 
El papel de los agentes institucionales debe ser permanente en estas zonas y no solamente a un accionar punitivo; la visión debe dirigirse a la capacitación y prevención.

El manejo de la gestión del riesgo debe realizarse de manera colaborativa e interinstitucional.

\section{Referencias}

Alcaldía de Villavicencio. 2016. Informe sobre violencia y actividades delictivas 2015- 2016.Villavicencio. Colombia.

Alfie-Cohen M, Castillo-Oropeza O. "Con el agua al cuello". Riesgo por inundación, vulnerabilidad socioambiental y gobernanza en el municipio de Cuautitlán. Quivera. Revista de Estudios Territoriales, 2016;18 (2):55-84

BANREP- Banco de la República. 2000. Actividad cultural. Colombia Prehispánica Regiones arqueológicas Instituto Colombiano de Antropología e Historia. http://www.banrepcultural.org/blaavirtual/arqueologia/prehisp/cp19.htm

BM- Banco Mundial. 2012. Análisis de la gestión del riesgo de desastres en Colombia: un aporte para la construcción de políticas públicas. Bogotá. Colombia.

Blaikie P, Terry C, Ian D, Ben W. 1996. Vulnerabilidad: el entorno social, político y económico de los desastre. Red de Estudios Sociales en Prevención de Desastres en América Latina. Colombia.

Borboa-Quintero M. Experiencia en Metodología Cualitativa: Historia de vida. Revista Ra ximhai, 2012;8(2):297-318.

Cardona OD. 1991. Evaluación de la Amenaza, la Vulnerabilidad y el Riesgo. Taller Regional de Capacitación para la Administración de Desastres. ONAD/PNUD/OPS/OEA. Bogotá. Colombia.

Carvajal-Escoba Y. Inundaciones en Colombia. ¿Estamos preparados para enfrentar la variabilidad y el cambio climático?. Revista Memorias, 2011; $9(16): 105-119$.

CIIFEN. Centro Internacional para la Investigación del Fenómeno de El Niño, 2015. Definición de Riesgo. http://www.ciifen.org/ index.php?option $=$ com content\&view $=$ category\&id=84\&layou $\underline{\mathrm{t}=\text { blog\&ltemid=111\&lang=es }}$

CEPAL- Comisión Económica para América Latina. 2011. La vulnerabilidad social y sus desafíos: una mirada desde América Latina. Santiago de Chile,http://repositorio.cepal.org/bitstream/handle/11362/4762/S0102116 es.pdf

Cohen B. "Urbanization in developing countries: current trends, future projections and key challenges for sustainability", Technologies in Society, 2006;28:

Cormacarena- Corporación para el Manejo del Área de la Macarena. 2008. Descripción afluentes de Villavicencio.

Da Cunha MP, Rodríguez VJ. Crecimiento urbano y movilidad en América Latina. Rev Latinoam Poblac, 2009;3( 4-5):27-64.

El Tiempo Periódico. Villavicencio vive una noche en medio de emergencia invernal. 29 de abril de 2016. http://www.eltiem- po.com/colombia/llano-7-dias/lluvias-en-el-meta/16576551 Publications.

Eraso ÁB, Icart IB, Gosálbez IP. El diseño del Análisis Cualitativo Multinivel: una aplicación práctica para el análisis de entrevistas. EMPIRIA. Revista de Metodología de las Ciencias Sociales, 2012;(24):15-44.

Faber-Benitez P. 2014 o 2010?. Terminología, traducción especializada y adquisición de conocimiento. La traducción en contextos especializados. Propuestas didácticas, Pp. 87-96.

Ferrari M. Análisis de vulnerabilidad y percepción social de las inundaciones en la ciudad de Trelew, Argentina. Cuad Geogr Rev Colomb Geogr, 2012;21(2):99-116.

La Federación Internacional de Sociedades de la Cruz Roja y de la Media Luna Roja. 2008. Desastres, gestión de desastres y vulnerabilidad. http://www.ifrc.org/es/introduccion/disastermanagement/sobre-desastres/que-es-un-desastre/que-es-lavulnerabilidad/ .

Llanes C. Los desastres nunca serán naturales. Revista INVI, 2003;18(47):41-53.

Leite M. 2011. Historias de Vida de Maestros y Maestras. La interminable construcción de las identidades: vida personal, trabajo y desarrollo personal. Tesis Doctoral, Universidad de Málaga.

IGUNNE- Instituto de Geografía Y Facultad de Humanidades. 2010. Resistencia, vulnerabilidad global y pobreza, Consideraciones conceptuales. Chaco. Argentina http://hum.unne.edu.ar/revistas/geoweb/Geo2/contenid/vulner6.htm

Patton QM. 1980. Qualitative Evaluations Methods. Beverly Hills. C.A. Sage.

POT- Plan de ordenamiento territorial vigencia 2015-2021 Villavicencio Meta- Alcaldía Villavicencio 2015. Colombia

Rausch J. 2011. De pueblo de frontera a Ciudad Capital, La historia de Villavicencio, Colombia, desde 1842. Banco de la República. Bogotá. Colombia.

Sedano-Cruz K, Carvajal-Escobar Y, Ávila Díaz Á. Análisis De Aspectos Que Incrementan El Riesgo De Inundaciones En Colombia. Revista Luna Azul, 2013;(37):219-238.

Torres M, Trujillo J. La ciudad y su dinámica. Orinoquía, 2014; 18(2): 7-10.

Trenberth K, Stepaniak D. "Indices of El Niño evolution". Journal of Climate, 2001;14:1697-1701.

UNISDR- Estrategia Internacional para la Reducción de Desastres de las Naciones Unidas. 2009. Terminología sobre Reducción de Riesgo de Desastres 2009 para los conceptos de Amenaza, vulnerabilidad y riesgo.

Hernando Castro: https://orcid.org/0000-0003-1299-0489 Francy Montealegre: https://orcid.org/0000-0001-7271-2697 